\title{
Comprehensive Microscopy of the Esophagus in Human Patients with Optical Frequency Domain Imaging
}

\section{Citation}

Suter, Melissa J., Benjamin J. Vakoc, Patrick S. Yachimski, Milen Shishkov, Gregory Y. Lauwers, Mari Mino-Kenudson, Brett E. Bouma, Norman S. Nishioka, and Guillermo J. Tearney. 2008. Comprehensive microscopy of the esophagus in human patients with optical frequency domain imaging. Gastrointestinal Endoscopy 68(4): 745-753. doi:10.1016/j.gie.2008.05.014

\section{Published Version}

doi:10.1016/j.gie.2008.05.014

\section{Permanent link}

http://nrs.harvard.edu/urn-3:HUL.InstRepos:32540816

\section{Terms of Use}

This article was downloaded from Harvard University's DASH repository, and is made available under the terms and conditions applicable to Other Posted Material, as set forth at http:// nrs.harvard.edu/urn-3:HUL.InstRepos:dash.current.terms-of-use\#LAA

\section{Share Your Story}

The Harvard community has made this article openly available.

Please share how this access benefits you. Submit a story.

Accessibility 


\title{
Comprehensive microscopy of the esophagus in human patients with optical frequency domain imaging
}

\author{
Melissa J. Suter, PhD, Benjamin J. Vakoc, PhD, Patrick S. Yachimski, MD, Milen Shishkov, \\ PhD, Gregory Y. Lauwers, MD, Mari Mino-Kenudson, MD, Brett E. Bouma, PhD, Norman S. \\ Nishioka, MD, and Guillermo J. Tearney, MD, PhD \\ Current affiliations: Wellman Center for Photomedicine, (M.J.S., B.J.V., M.S., B.E.B., G.J.T.), \\ Department of Dermatology (M.J.S., B.J.V., M.S., B.E.B.), Gastrointestinal Unit (P.S.Y., N.S.N.), \\ and Department of Pathology (G.Y.L., M.M.K., G.J.T.), Harvard Medical School, Massachusetts \\ General Hospital Boston, Massachusetts, USA
}

\begin{abstract}
Background-Optical coherence tomography (OCT) is a cross-sectional, high-resolution imaging modality that has been shown to accurately differentiate esophageal specialized intestinal metaplasia (SIM) from gastric cardia at the squamocolumnar junction (SCJ) and diagnose high-grade dysplasia and intramucosal carcinoma in patients with SIM. The clinical utility of OCT has been limited, however, by its inability to acquire images over large areas.
\end{abstract}

Objective-The aim of this study was to use recently developed high-speed OCT technology, termed optical frequency domain imaging (OFDI), and a new balloon-centering catheter $(2.5 \mathrm{~cm}$ diameter) to demonstrate the feasibility of large area, comprehensive optical microscopy of the entire distal esophagus $(\sim 6.0 \mathrm{~cm})$ in patients.

Design-A pilot feasibility study.

Setting-Massachusetts General Hospital.

Patients-Twelve patients undergoing routine EGD.

Results-Comprehensive microscopy of the distal esophagus was successfully performed in 10 patients with the OFDI system and balloon catheter. There were no complications resulting from the imaging procedure. Volumetric data sets were acquired in less than 2 minutes. OFDI images at the SCJ showed a variety of microscopic features that were consistent with histopathologic findings, including squamous mucosa, cardia, SIM with and without dysplasia, and esophageal erosion.

Limitations-Inability to obtain direct correlation of OFDI data and histopathologic diagnoses.

Conclusions-Comprehensive volumetric microscopy of the human distal esophagus was successfully demonstrated with OFDI and a balloon-centering catheter, providing a wealth of detailed information about the structure of the esophageal wall. This technique will support future studies to compare OFDI image information with histopathologic diagnoses.

\section{(C) 2008 by the American Society for Gastrointestinal Endoscopy}

Reprint requests: Guillermo J. Tearney, MD, PhD, Department of Pathology, Harvard Medical School, Massachusetts General Hospital and Wellman Center for Photomedicine, 40 Blossom St, BAR 703, Boston, MA 02114.

DISCLOSURE

The authors report that there are no disclosures relevant to this publication. This work was supported by National Institutes of Health grant No. R01CA103769 and by the Center for Integration of Medicine and Innovative Technology (development of the imaging platform). 
The pathogenesis of esophageal adenocarcinoma is thought to involve a stepwise progression from gastroesophageal reflux to specialized intestinal metaplasia (SIM) of the esophagus, lowgrade dysplasia (LGD), high-grade dysplasia (HGD), and finally adenocarcinoma. ${ }^{1}$ Current management guidelines therefore promote screening individuals with GERD for esophageal SIM and regular surveillance of patients with SIM to increase the chances of detecting neoplasia early in the disease process. ${ }^{2}$ Because endoscopy alone may not be sufficient to distinguish Barrett's esophagus (BE) from irregular z-lines or to identify dysplasia and intramucosal carcinoma (IMC), current guidelines include systematic, but unguided, biopsy, which is prone to significant sampling errors. ${ }^{3-6}$ The deficiencies of random biopsy are highlighted by the poor positive predictive value of endoscopic screening, estimated to be as low as $34 \%,{ }^{3}$ and by data demonstrating that many patients with BE diagnosed with HGD harbor occult adenocarcinoma else-where in the esophagus. ${ }^{2,7,8}$ The limitations of the current random biopsy practice therefore suggests an important role for a method that can accurately and comprehensively evaluate the entire distal esophagus on the microscopic scale.

Optical coherence tomography (OCT) is a noncontact optical imaging modality that generates cross-sectional images of tissues with a resolution $(<10 \mu \mathrm{m})$ comparable to that of low-power $(4 \times)$ brightfield microscopy, which is sufficient for detecting microscopic tissue architecture. 9-14 The potential of OCT for diagnosing SIM has been established with prospective studies showing sensitivities ranging from $81 \%$ to $97 \%$ and specificities from $57 \%$ to $92 \% .{ }^{15,16}$ Dysplasia grading is also possible with this technology. Reported sensitivities for detecting HGD/IMC range from $54 \%$ to $83 \%$ with corresponding specificities of $72 \%$ to $75 \% .{ }^{17,18}$ The potential of OCT for upper GI diagnosis has not been realized clinically, however, because of the point sampling nature of the technology, making it subject to the same sampling errors as random biopsy. A class of second-generation OCT instruments have recently been developed that may change this outlook. Among them, optical frequency domain imaging (OFDI) ${ }^{19,20}$ produces images that are identical to those obtained by OCT, but at a rate that is approximately 2 orders of magnitude faster. In combination with a recently developed balloon-centering optical catheter, ${ }^{21}$ OFDI makes it possible to comprehensively image the entire distal esophagus in a time ( $<2$ minutes, $50 \mu \mathrm{m}$ pitch) that is acceptable for an endoscopic procedure. OFDI has been demonstrated previously in the esophagus of normal swine in vivo. ${ }^{21}$ In this article, we present our first experience with this technique in human patients.

\section{EXPERIMENTAL METHODS}

\section{Patient enrollment}

Patients undergoing a routine EGD for screening and surveillance of BE at Massachusetts General Hospital were enrolled in this study from November 2006 to July 2007. The study exclusion criteria included all individuals with bleeding issues, with a known diagnosis of an esophageal or gastric mass, or patients under increased emotional stress. Potential study participants were sent a letter informing them of the study procedure before the day of the scheduled EGD. On the day of the scheduled EGD, patients were further informed about the study and if they expressed an interest in participating, informed consent was obtained (28\%). The study protocol and consent process were approved by the Partners Internal Review Board (Institutional Review Board No. 2006-P-00203).

\section{OFDI and balloon centering optical catheter}

The technical details of the OFDI imaging system and balloon catheter have been described previously. ${ }^{19-22}$ The balloon catheter had an inflated diameter of $25 \mathrm{~mm}$, an overall inflated length of $7 \mathrm{~cm}$, and additionally contained a rapid exchange guidewire provision at its most distal aspect (Fig. 1A). Supporting structures were added to the proximal end of the balloon to stabilize the inner sheath within the inflated balloon (Fig. 1A). Helical scanning of the distal 
optics through the length of the balloon was accomplished by the use of an optical rotary junction and pull-back device, fabricated in our laboratory (Fig. 1B). The OFDI system constructed for the purpose of this study was capable of acquiring, processing, and displaying image data at a sustained A-line rate of $40 \mathrm{kHz}$, corresponding to a complete esophageal crosssectional imaging speed of 9.8 frames/second (frame size: $2048[\mathrm{r}] \times 4096[\varphi]$ ). With these specifications, volumetric microscopy data from 6-cm (z) lengths of the distal esophagus were obtained in 2 minutes, with image resolutions of $20 \mu \mathrm{m}(\varphi) \times 8 \mu \mathrm{m}(\mathrm{r}) \times 50 \mu \mathrm{m}(\mathrm{z})$. During imaging, all raw data was continuously stored in a RAID high-speed data storage device (Signatec Inc, Newport Beach, Calif) while a subset of data was periodically (approximately 4 frames/second) processed, circularized, and displayed in real time for immediate visualization by the physician and research team. Additionally, encoder signals from the rotary junction and pull-back device motors were simultaneously recorded to archive the circumferential and longitudinal location of the sampling location throughout the helical scan. The raw data for a typical volumetric OFDI data set, scanned over an esophageal length of 6 $\mathrm{cm}$, comprised $38 \mathrm{~GB}$.

During the process of developing the system and catheter described above, patients $(\mathrm{n}=32)$ were imaged as the system and catheter were being refined and before the designs were fixed. The OFDI imaging system used in these preliminary studies acquired images at an A-line rate of $5 \mathrm{kHz}$, corresponding to 1.4 frames/second (frame size: $1920[\mathrm{r}] \times 3460[\varphi]$ ). In the results section, we show data from these early systems that are clinically relevant (Figs. 2A and 2B). Statistics on imaging efficacy are only presented from cases $(n=12)$ that were imaged with the final system and catheter.

\section{Imaging procedure}

An upper endoscopy was performed on each of the study participants. Throughout the procedure, the patient's blood pressure, pulse, and oxygen saturation were monitored continuously. The distance to the squamocolumnar junction (SCJ) was measured with the endoscope to determine the insertion length for the OFDI imaging catheter. After endoscopic evaluation and digitization of representative videoendoscopic images, a guidewire was passed through the auxiliary endoscope channel into the stomach. The endoscope was with-drawn, the balloon catheter was advanced along the guidewire to the SCJ, and the guidewire was removed. The balloon was then inflated and an initial scout scan, obtained by acquiring crosssectional images at the distal and proximal aspects of the balloon, was performed to ensure correct balloon placement. If OFDI images of stomach and squamous mucosa were not observed in the distal and proximal scout scans, respectively, the balloon was deflated and repositioned until correct placement was confirmed. The optical rotary junction and pullback were then initialized, and helical cross-sectional images were obtained over an esophageal length of up to $6.5 \mathrm{~cm}$.

\section{Biopsy acquisition}

At completion of the image acquisition, the OFDI balloon catheter was deflated and removed from the patient. Biopsy specimens were subsequently obtained at the gastroesophageal junction. For patients with endoscopically apparent SIM, random 4-quadrant biopsy specimens were obtained at 2-cm intervals, as per the standard of care. Specimens were processed in the usual manner. Histopathologic diagnoses were rendered by the GI pathologists in the Massachusetts General Hospital pathology department.

\section{Image processing and interpretation}

After the procedure all images were reconstructed with radial and circumferential coordinates displayed in rectangular format from the raw OFDI data. To obtain longitudinal sections and 3-dimentional representations of the data sets, the individual frames were registered to the 
rotary junction and pullback motor encoder data. Additionally, the images were aligned with use of a snake algorithm ${ }^{23}$ so that the surface of the balloon appeared at a constant distance from the imaging optics. Longitudinal images and 3-dimensional representations were prepared with ImageJ 1.38w (National Institute of Health, Bethesda, Md) and Osirix 2.75 (University Hospital of Geneva, Geneva, Switzerland) software, respectively. Images are displayed by use of an inverse gray-scale lookup table. For purposes of display only, to conform to figure size constraints, the radial and circumferential coordinates are not displayed on the same scale for figures depicting entire circumferential cross-sections. An experienced OFDI reader (G. J. T) evaluated the volumetric data sets obtained from each study participant and rendered a diagnosis on the basis of previously defined and validated criteria (Table 1) for diagnosis of esophageal conditions. ${ }^{15,16,18}$ In this study, the biopsy sites could not be precisely registered to the OFDI volume data sets because the OFDI images were acquired before the biopsy procedure. Therefore, for display purposes, representative OFDI sections were chosen that corresponded to the histopathologic diagnoses or the appearance of the histopathologic slides at low-power magnification.

\section{Capsule Summary}

\section{What is already known on this topic}

- Optical frequency domain imaging (OFDI) produces images that are identical to those obtained by optical coherence tomography, but at a faster rate.

\section{What this study adds to our knowledge}

- In 10 of 12 patients undergoing routine EGD, comprehensive microscopy of the distal esophagus was successfully performed without complication by using the OFDI system and a balloon optical catheter.

- Volumetric data sets were acquired in less than 2 minutes.

\section{RESULTS}

A total of 12 patients were enrolled in the study after the system and balloon catheter designs were fixed. OFDI imaging was successfully conducted in 10 patients. Imaging was not successful in 2 of the patients because of an imaging system malfunction. The average length of pullback was $5.70 \mathrm{~cm} \pm 0.97 \mathrm{~cm}$ (SD). Of the 10 cases in which OFDI imaging was performed, the SCJ was completely visualized in $8(80 \%)$ cases. In $2(20 \%)$ cases, only a small segment of the SCJ was visible because of improper positioning and movement of the balloon catheter on inflation. A loss of evaluatable image data in individual frames resulted from inadequate contact of the balloon catheter or from the presence of mucus. These artifacts were observed, on average, in $0.37 \% \pm 0.79 \%$ (SD) of the total tubular esophageal surface area per patient. Study participation, including insertion and inflation of the OFDI balloon catheter, OFDI imaging, and removal of the catheter, added less than 8 minutes ( $\sim 4$ minutes on average) to the length of time required for the EGD procedure.

\section{Normal esophagus and cardia}

Figure 2 depicts cross-sectional images that satisfy the OCT criteria for normal esophagus and cardia. ${ }^{15}$ Figures $2 \mathrm{~A}$ and $2 \mathrm{~B}$ were obtained from an 87 -year-old female patient with a history of GERD, and Figures 2C and 2D were obtained from a 61-year-old man with mild dysphagia. The layered architecture of normal squamous mucosa (Figs. 2A and 2B) can be clearly identified. In contrast to previous work that was conducted with a noncontact probe, images acquired with the balloon-centering catheter show the lamina propria and muscularis mucosa as one single backscattering layer (Fig. 2B). Images of cardia demonstrated the broad, regular 
architecture, "pit and crypt" appearance, high superficial reflectivity, and decreased image penetration (Figs. 2C and 2D) seen in prior work. ${ }^{15}$

\section{Ulcerated squamous mucosa}

Endoscopy, OFDI, and histologic images taken from a 43-year-old woman with no history of BE are shown in Figure 3. During endoscopy (Fig. 3A) it was apparent that the squamous mucosa was highly irregular. OFDI images revealed that there were no signs of SIM; however, the superficial layers of the squamous epithelium were clearly eroded (Fig. 3C, arrow). The histologic findings (Fig. 3B) were consistent with the OFDI results, demonstrating inflamed squamoglandular junctional mucosa with reflux esophagitis and erosion but no signs of SIM.

\section{Specialized intestinal metaplasia}

The images in Figure 4 were obtained from a 62-yearold male patient with a history of BE. On endoscopic examination, the patient had an irregular appearing SCJ (Fig. 4A). Biopsy results confirmed SIM without evidence of dysplasia (Fig. 4B). A transverse cross-sectional OFDI image (Fig. 4C) obtained at the SCJ shows regions of both squamous epithelium (Fig. 4C, red arrow, and Fig. 4D) and areas that lacked both squamous and cardiac architecture and that exhibited heterogeneous backscattering and an irregular surface, suggestive of SIM (Fig. 4C, blue arrows, and Fig. 4E). Likewise, a longitudinal image spanning the entire $5.05 \mathrm{~cm}$ acquisition length demonstrates an abrupt transition from the squamous mucosa to a region that is consistent with SIM (arrow, Fig. 4F). The longitudinal extent of the BE segment was found to be approximately $7 \mathrm{~mm}$. The lamina propria and submucosa of the squamous epithelium appear jagged in the longitudinal image, likely from motion artifacts caused by peristalsis.

Figure 5 demonstrates endoscopic, OFDI, and histology images obtained from a 56-year-old woman with an irregular SCJ. A fly-through view of the maximum intensity projection (MIP) ${ }^{24}$ of the entire OFDI data set, shown in Figure 5B, highlights the irregular SCJ. A crosssectional OFDI image acquired at the SCJ shows a segment of squamous mucosa interspersed with OFDI-apparent SIM (Fig. 5C). The longitudinal reconstruction (Fig. 5E) indicates that the length of the BE segment is $12 \mathrm{~mm}$. The pathology results from a biopsy specimen obtained at the SCJ (Fig. 5D) showed only squamoglandular junctional mucosa with chronic inflammation and no signs of SIM. The discrepancy between OFDI and biopsy results in this case could be due to biopsy sampling error or misdiagnosis of the OFDI images. ${ }^{15}$

\section{Dysplasia}

Like the modified Haggitt criteria for histopathologic scoring of dysplasia in esophageal SIM, ${ }^{25}$ poor surface maturation and irregular gland architecture have also been demonstrated to provide a basis for grading dysplasia in OCT. ${ }^{18}$ Figure 6 was obtained from a 45 -year-old man with a previous history of BE and focal HGD. A 2-cm BE segment was noted during endoscopy (Fig. 6A). Examination of the OFDI data set revealed regions consistent with squamous mucosa, SIM, LGD, and HGD (Fig. 6C). OFDI evidence of SIM without dysplasia can be seen in an expanded portion of the cross-sectional image (Fig. 6D), where good surface maturation is seen, with the surface reflectivity being lower than that of the subsurface. ${ }^{18}$ Conversely, the segment of mucosa in Figure 6E is indicative of SIM with LGD and HGD. Figure 6E demonstrates poor surface maturation, manifested by high backscattering at the surface, ${ }^{18}$ and the presence of dilated and mildly distorted glands and ducts in the mucosa. Histopathologic findings from a biopsy specimen obtained at this esophageal level showed glandular mucosa with SIM, acute inflammation, and focal epithelial atypia consistent with HGD. 


\section{DISCUSSION}

Our initial experience with balloon catheter-based OFDI indicates that comprehensive imaging of the microscopic architecture of the distal esophagus is feasible and safe. Furthermore, our results suggest that comprehensive OFDI may enable the visualization of disease that cannot be seen by endoscopy and subsequent biopsy. By screening the distal esophagus to identify suspect regions that contain disease that cannot be recognized by videoendoscopy, this technology may be used to guide biopsy and minimize sampling error associated with the random biopsy technique.

However, to realize the potential of this technology, additional research must be conducted to improve our understanding of the images. In this article, we have attempted to interpret our OFDI images by using criteria developed from OCT images obtained with a noncontact linearly scanning probe. ${ }^{9}$ When in contact with the esophageal wall, the balloon catheter necessarily compressed the mucosa, making it difficult to visualize surface topology, which is one feature that has been used to diagnose SIM. ${ }^{15}$ Furthermore, studies conducted to investigate the effects of compression on OCT images have shown that the images change significantly with increasing pressure ${ }^{26}$ The degree to which mucosal compression by the balloon affects the accuracy of previously developed OCT diagnostic criteria applied to OFDI images is unknown. Finally, we currently do not understand the intraobserver or interobserver diagnostic variability for balloon-OFDI imaging, and we do not have information on the reproducibility of the findings.

A study to compare balloon-based OFDI and histopathologic diagnoses would be valuable for answering these questions. In our current study, we were not able to obtain a one-to-one correlation of OFDI data with histopathologic diagnoses because we could not register the 3dimensional data set with the biopsy location. A possible solution to this problem would be to reimage the esophagus after biopsy. After coregistration of the two OFDI volumes, the biopsy locations seen on the second data set could then be used to identify the image regions in the first data set that correspond to the biopsy sites. Difficulties with this approach include the presence of blood, which could attenuate the optical signal and the additional time required for the experimental procedure. Another possibility is some form of esophageal marking applied when images of interest are identified after the OFDI scan and while the balloon is still in place. Biopsy specimens could then be acquired at the marked location after withdrawal of the balloon. Adequate targeting can be evaluated by performing OFDI of the biopsy specimen to ensure that it contains the same images that were obtained in vivo. Development of such a technique would also be beneficial clinically because it would provide the foundation for a guided biopsy platform.

Other artifacts such as poor balloon contact with the esophagus and peristalsis affected image quality. In some individuals, especially those with large hiatal hernias, tissue contact with the balloon surface was not maintained throughout the full 360-degree, $\sim 6$-cm imaging window. Although OFDI does not require a transducing medium, mucus and blood between the balloon surface and the esophageal wall sometimes decreased the OFDI signal, reducing the clarity of the images. Future development of a catheter with a variable inflation diameter could provide adequate tissue contact while minimizing excessive tissue compression. Peristalsis made it difficult to reconstruct longitudinal data sets with fidelity because the balloon typically oscillated along the longitudinal extent of the esophagus. Increased imaging speed ${ }^{27}$ and proper contact between the balloon and the esophageal wall could minimize this artifact.

Although comprehensive evaluation of the entire distal esophagus with the OFDI ballooncatheter holds the promise of reducing the sampling error associated with BE screening and surveillance, it introduces challenges associated with the manipulation, assessment, and storage 
of very large digital data files. In our study, storage requirements approached $40 \mathrm{~GB}$ for a single volumetric pullback. Additionally, because a single $6-\mathrm{cm}$ pullback contains approximately 1200 cross-sectional images, where each un-compressed image spanned a dimension of 7168 $\times 7168$ pixels (49 MB per image), rigorous assessment and interpretation of the acquired data were time consuming and laborious. The development of automated or semiautomated computer-aided detection and diagnostic algorithms will therefore be a high priority for future research. One possibility is that the entire volume will be scanned with OFDI, and through the use of pattern recognition algorithms, the computer will automatically select subsets of the volume data set that are most likely to contain the severest disease. These subsets will then be presented to the physician in 2- or 3-dimensional image format. Such algorithms would significantly decrease the time required to evaluate the OFDI data sets, facilitating diagnosis in the endoscopy suite at the time of the procedure.

\section{Abbreviations}

BE, Barrett's esophagus; HGD, high-grade dysplasia; LGD, low-grade dysplasia; IMC, intramucosal carcinoma; MIP, maximum intensity projection; OCT, optical coherence tomography; OFDI, optical frequency domain imaging; SCJ, squamocolumnar junction; SIM, specialized intestinal metaplasia.

\section{ACKNOWLEDGMENTS}

We thank William Puriceilli for his assistance in all clinical aspects of this research.

\section{REFERENCES}

1. Lagergren J, Bergstrom R, Lindgren A. Symptomatic gastroesophageal reflux and a risk factor for esophageal adenocarcinoma. N Engl J Med 1999;340:825-831. [PubMed: 10080844]

2. Incarbone R, Bonavina L, Saino G, et al. Outcome of esophageal adenocarcinoma detected during endoscopic biopsy surveillance for Barrett's esophagus. Surg Endosc 2002;16:263-266. [PubMed: 11967675]

3. Eloubeidi MA, Provenzale D. Does this patient have Barrett's esophagus? The utility of predicting Barrett's esophagus at the index endoscopy. Am J Gastroenterol 1999;94:937-943. [PubMed: 10201460]

4. Dulai GS. Surveying the case for surveillance. Gastroenterology 2002;122:820-823. [PubMed: 11875016]

5. Falk GW, Chittajallu R, Goldblum JR, et al. Surveillance of patients with Barrett's esophagus for dysplasia and cancer with balloon cytology. Gastroenterology 1997;112:1787-1797. [PubMed: 9178668]

6. Streitz JM, Andrews CW, Ellis FH. Endoscopic surveillance of Barrett's esophagus: does it help? J Thorac Cardiovasc Surg 1993;105:383-387. [PubMed: 8445916]

7. Peters JH, Clark GWB, Ireland AP, et al. Outcome of adenocarcinoma arising in Barrett's esophagus in endoscopically surveyed and nonsurveyed patients. J Thorac Cardiovascular Surg 1994;108:813822 .

8. Konda VJA, Ross AS, Ferguson MK, et al. Is the risk of concomitant invasive esophageal cancer in high-grade dysplasia in Barrett's esophagus overestimated? Clin Gastroenterol Hepatol 2008;6:159_ 164. [PubMed: 18096439]

9. Bouma BE, Tearney GJ, Compton CC, et al. High-resolution imaging of the human esophagus and stomach in vivo using optical coherence tomography. Gastrointest Endosc 2000;51:467-474. [PubMed: 10744824]

10. Sergeev AM, Gelikonov VM, Gelikonov GV, et al. In vivo endoscopic OCT imaging of precancer and cancer states of human mucosa. Optics Express 1997;1:432-440. [PubMed: 19377567]

11. Sivak MV Jr, Kobayashi K, Izatt JA, et al. High-resolution endoscopic imaging of the GI tract using optical coherence tomography. Gastrointest Endosc 2000;51:474-479. [PubMed: 10744825] 
12. Tearney GJ, Brezinski ME, Bouma BE, et al. In vivo endoscopic optical biopsy with optical coherence tomography. Science 1997;276:2037-2039. [PubMed: 9197265]

13. Hsiung P-L, Pantanowitz L, Aguirre AD, et al. Ultrahigh-resolution and 3-dimensional optical coherence tomography ex vivo imaging of the large and small intestines. Gastrointest Endosc 2005;62:561-574. [PubMed: 16185971]

14. Chen Y, Aguirre AD, Hsiung P-L, et al. Ultrahigh resolution optical coherence tomography of Barrett's esophagus: preliminary descriptive clinical study correlating images with histology. Endoscopy 2007;39:599-605. [PubMed: 17611914]

15. Evans JA, Poneros JM, Bouma BE, et al. Identifying intestinal metaplasia at the squamocolumnar junction using optical coherence tomography. Gastrointest Endosc 2007;65:50-56. [PubMed: 17137858]

16. Poneros JM, Brand S, Bouma BE, et al. Diagnosis of specialized intestical metaplasia by optical coherence tomography. Gastroenterology 2001;120:7-12. [PubMed: 11208708]

17. Isenberg G, Sivak MV Jr, Chak A, et al. Accuracy of endoscopic optical coherence tomography in the detection of dysplasia in Barrett's esophagus: a prospective, double-blinded study. Gastrointest Endosc 2005;62:825-831. [PubMed: 16301020]

18. Evans JA, Poneros JM, Bouma BE, et al. Optical coherence tomography to identify intramucosal carcinoma and high-grade dysplasia in Barrett's esophagus. Clin Gastroenterol Hepatol 2006;4:3843. [PubMed: 16431303]

19. Yun SH, Tearney GJ, Vakoc BJ, et al. Comprehensive volumetric optical microscopy in vivo. Nat Med 2006;12:1429-1433. [PubMed: 17115049]

20. Yun SH, Tearney GJ, de Boer JF, et al. High-speed optical frequencydomain imaging. Optics Express 2003;11:2953-2963. [PubMed: 19471415]

21. Vakoc BJ, Shishkov M, Yun SH, et al. Comprehensive esophageal microscopy by using optical frequency-domain imaging. Gastrointest Endosc 2007;65:898-905. [PubMed: 17383652]

22. Li XD, Boppart SA, Van Dam J, et al. Optical coherence tomography: advanced technology for the endoscopic imaging of Barrett's esophagus. Endoscopy 2000;32:921-930. [PubMed: 11147939]

23. Kass M, Witkin A, Terzopoulos D. Snakes: active contour models. Int J Comput Vis 1988;1:321331.

24. Wallis JW, Miller TR, Lerner CA, et al. Three-dimensional display in nuclear medicine. IEEE Trans Med Imaging 1989;8:297-303. [PubMed: 18230529]

25. Montgomery E, Bronner MP, Goldblum JR, et al. Reproducibility of the diagnosis of dysplasia in Barrett esophagus: a reaffirmation. Hum Pathol 2001;32:368-378. [PubMed: 11331953]

26. Westphal V, Rollins AM, Willis J, et al. Correlation of endoscopic optical coherence tomography with histology in the lower-GI tract. Gastrointest Endosc 2005;61:537-546. [PubMed: 15812406]

27. Oh WY, Yun SH, Vakoc BJ, et al. Ultrahigh-speed optical frequency domain imaging and application to laser ablation monitoring. Appl Phys Lett 2006;88:103902.1-3 

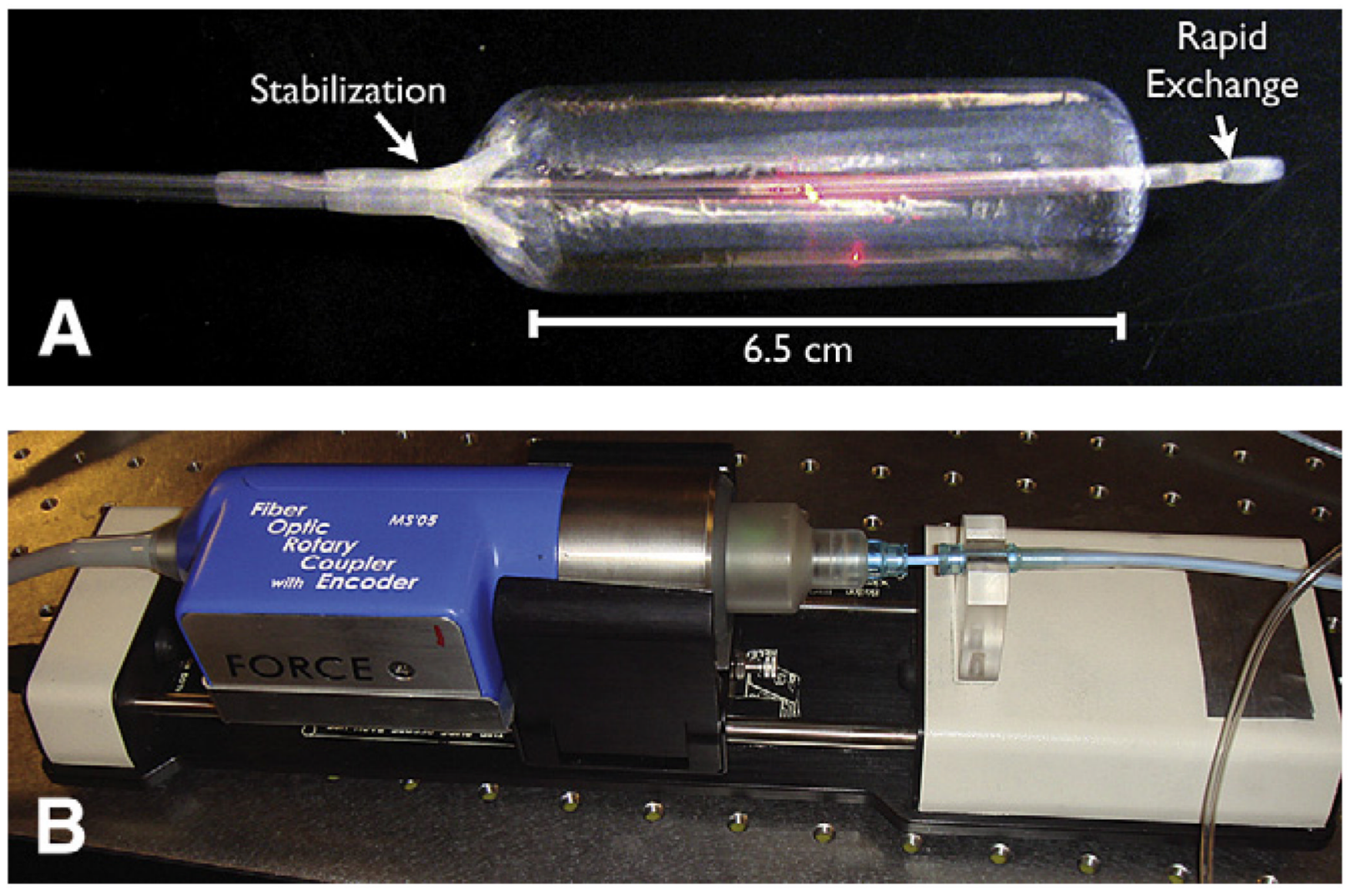

Figure 1.

A, Balloon catheter with proximal stabilization device and distal rapid guidewire exchange provision. The balloon acts to dilate the esophageal lumen and center the inner optical core. The inflation diameter of the balloon is $25 \mathrm{~mm}$ and the imaging window is $6.5 \mathrm{~cm}$. The inner optical core ( $1 \mathrm{~mm}$ in diameter) is contained inside a sheath that allows the core to move (rotate and translate) independently of the balloon catheter. B, Optical rotary junction mounted on the pullback tray and connected to the proximal portion of the imaging catheter. 

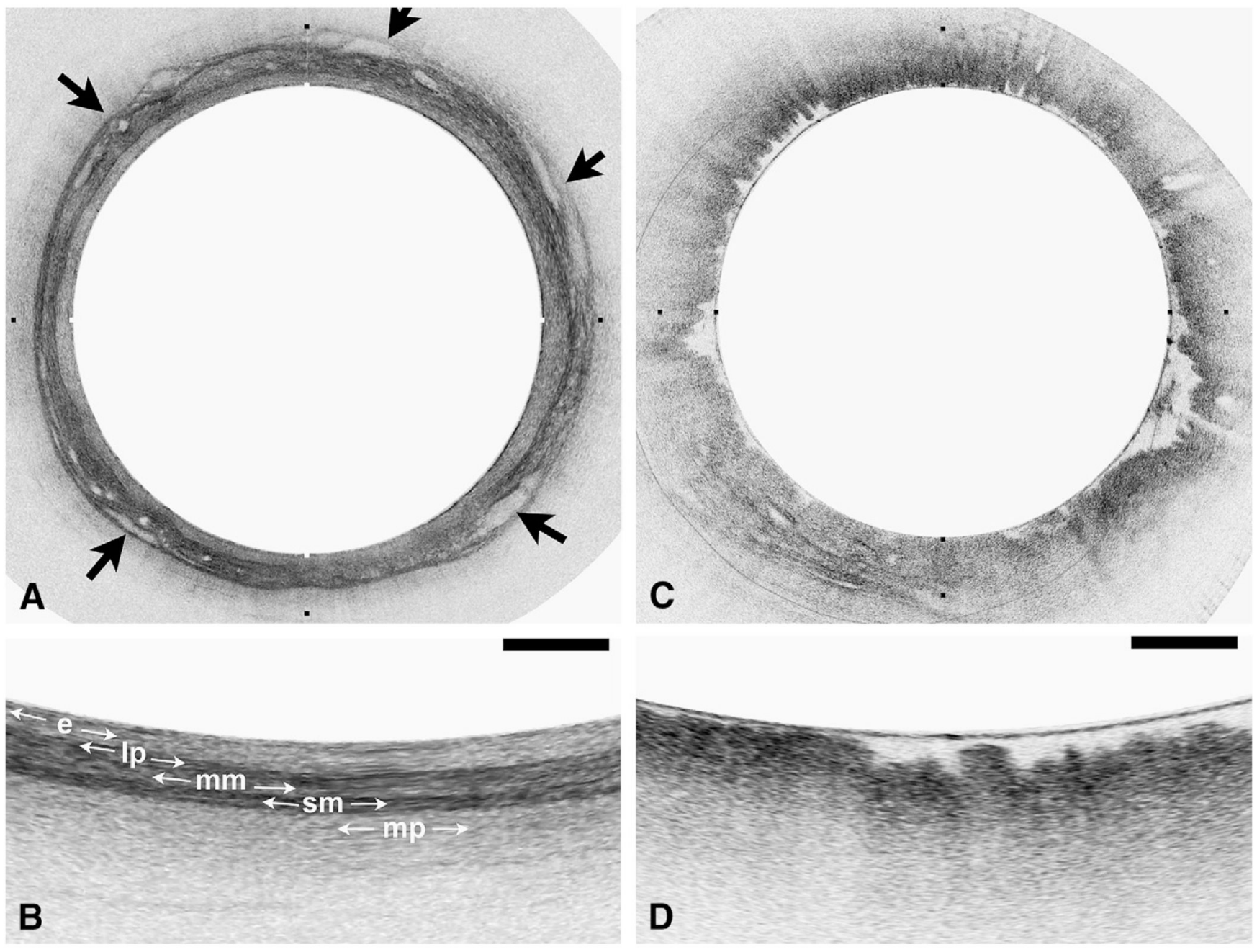

Figure 2.

OFDI images obtained from patients with a normal-appearing stomach and esophagus by endoscopy. A, OFDI image of squamous mucosa. B, Expanded view of $\mathbf{A}$ demonstrates a layered appearance, including the epithelium (e), lamina propria $(l p)$, muscularis mucosa $(\mathrm{mm})$, submucosa $(\mathrm{sm})$, and muscularis propria $(\mathrm{mp})$. Vessels are clearly identified in the submucosa (arrows). C, OFDI image of gastric cardia. D, Expanded view of $\mathbf{C}$ demonstrates vertical pit and crypts, regular, broad architecture, high surface backscattering, and diminished image penetration. Tick marks in $\mathbf{A}$ and $\mathbf{C}$ and scale bars in $\mathbf{B}$ and $\mathbf{D}$ represent $1 \mathrm{~mm}$. 

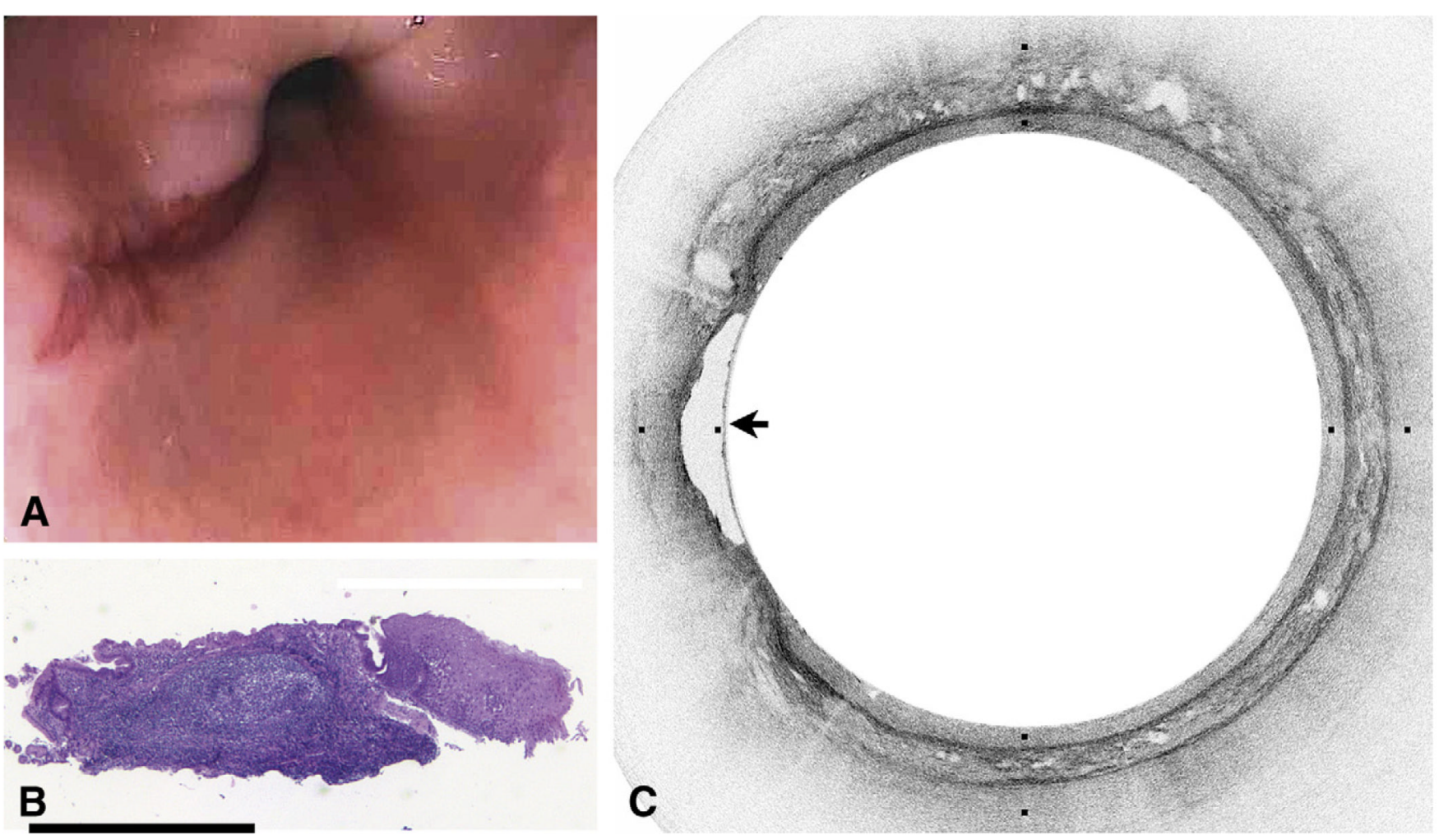

Figure 3 .

Esophageal erosion. A, Videoendoscopy image reveals the presence of a localized injury. B, Histopathologic image of the biopsy specimen demonstrates esophageal erosion and chronic inflammation (H\&E, orig. mag. $\times 2$ ). $\mathbf{C}$, Cross-sectional OFDI image traversing the injury shows a region where the squamous epithelial layer is absent (arrow). Tick marks in $\mathbf{C}$ and scale bar in $\mathbf{B}$ represent $1 \mathrm{~mm}$. 

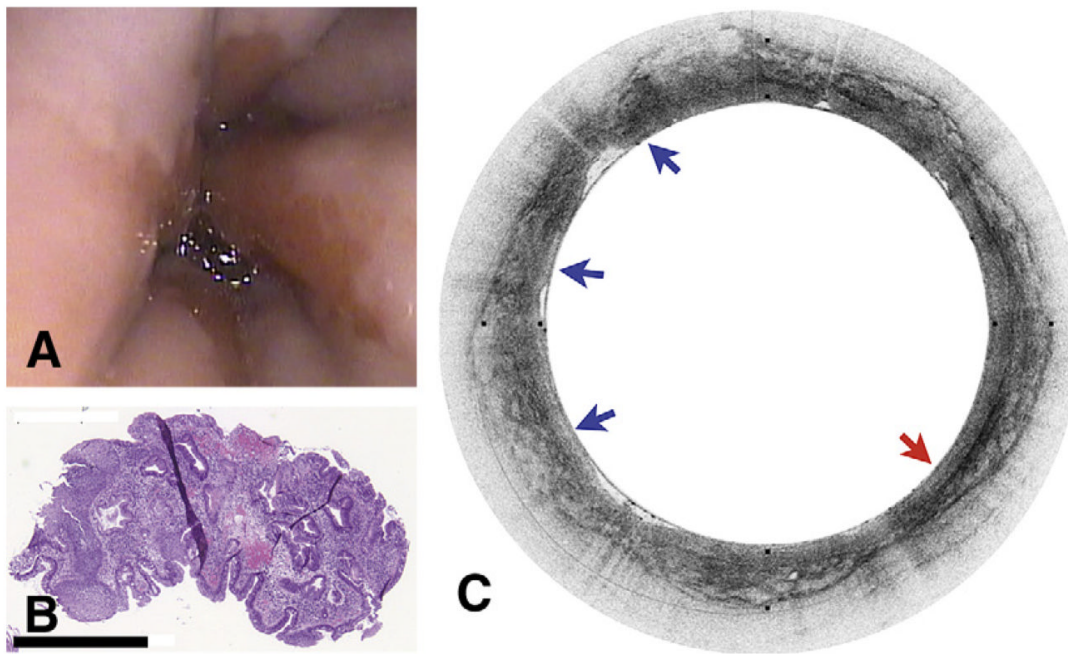

D
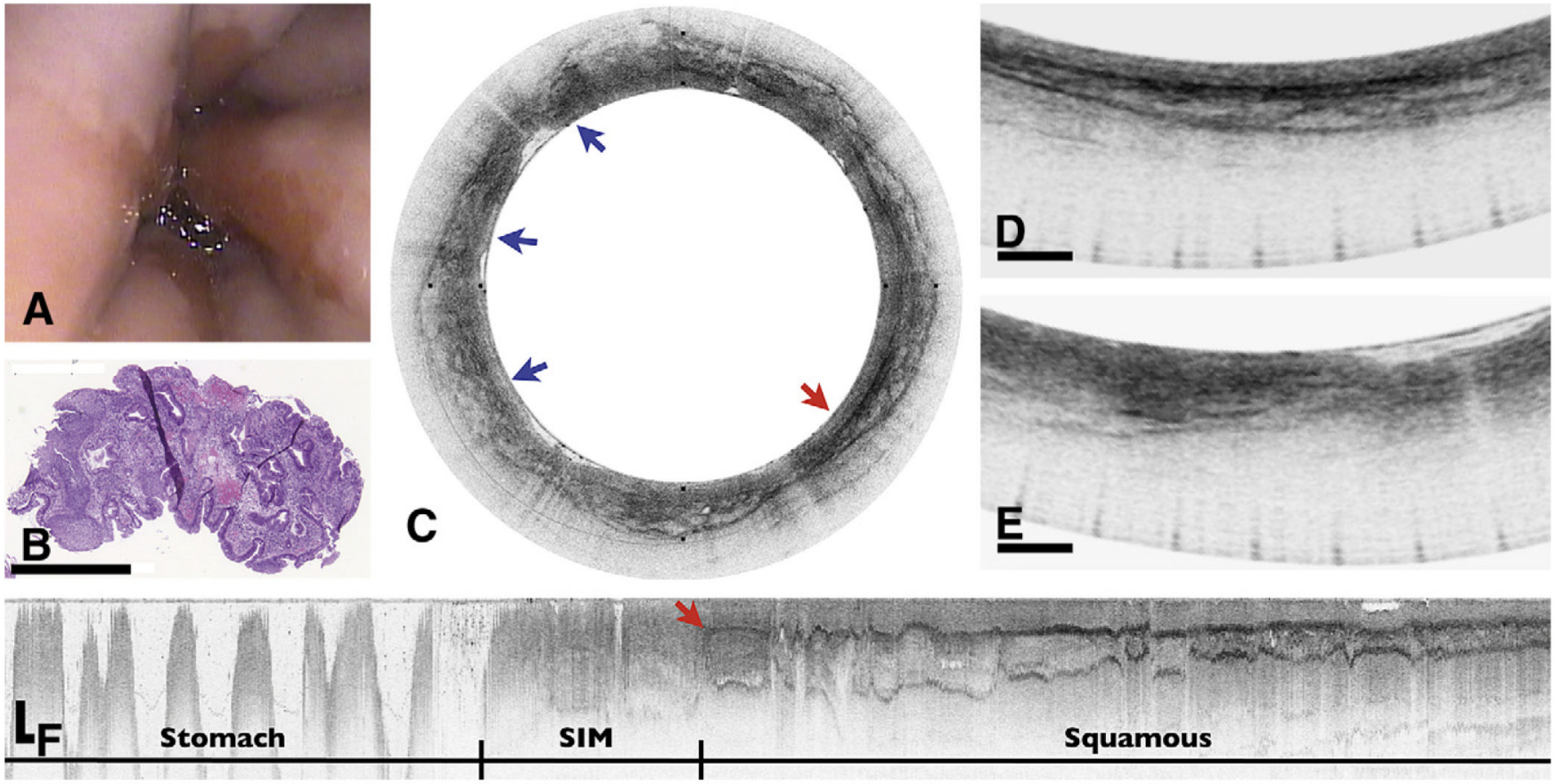

\section{Figure 4.}

Barrett's esophagus. A, Videoendoscopic image demonstrates an irregular SCJ. B, Histopathologic image of a biopsy specimen obtained from the SCJ demonstrates SIM without dysplasia (H\&E, orig. mag. $\times 2$ ). C, Cross-sectional OFDI image reveals both the normal layered appearance of squamous mucosa (red arrow, expanded in D) and tissue that satisfies the OCTcriteria for SIM (blue arrows, expanded in E). F, Longitudinal section across the gastroesophageal junction shows the transition from squamous mucosa to SIM to cardia. The length of the BE segment is $7 \mathrm{~mm}$ in this OFDI reconstruction. Scale bars and tick marks represent $1 \mathrm{~mm}$. 


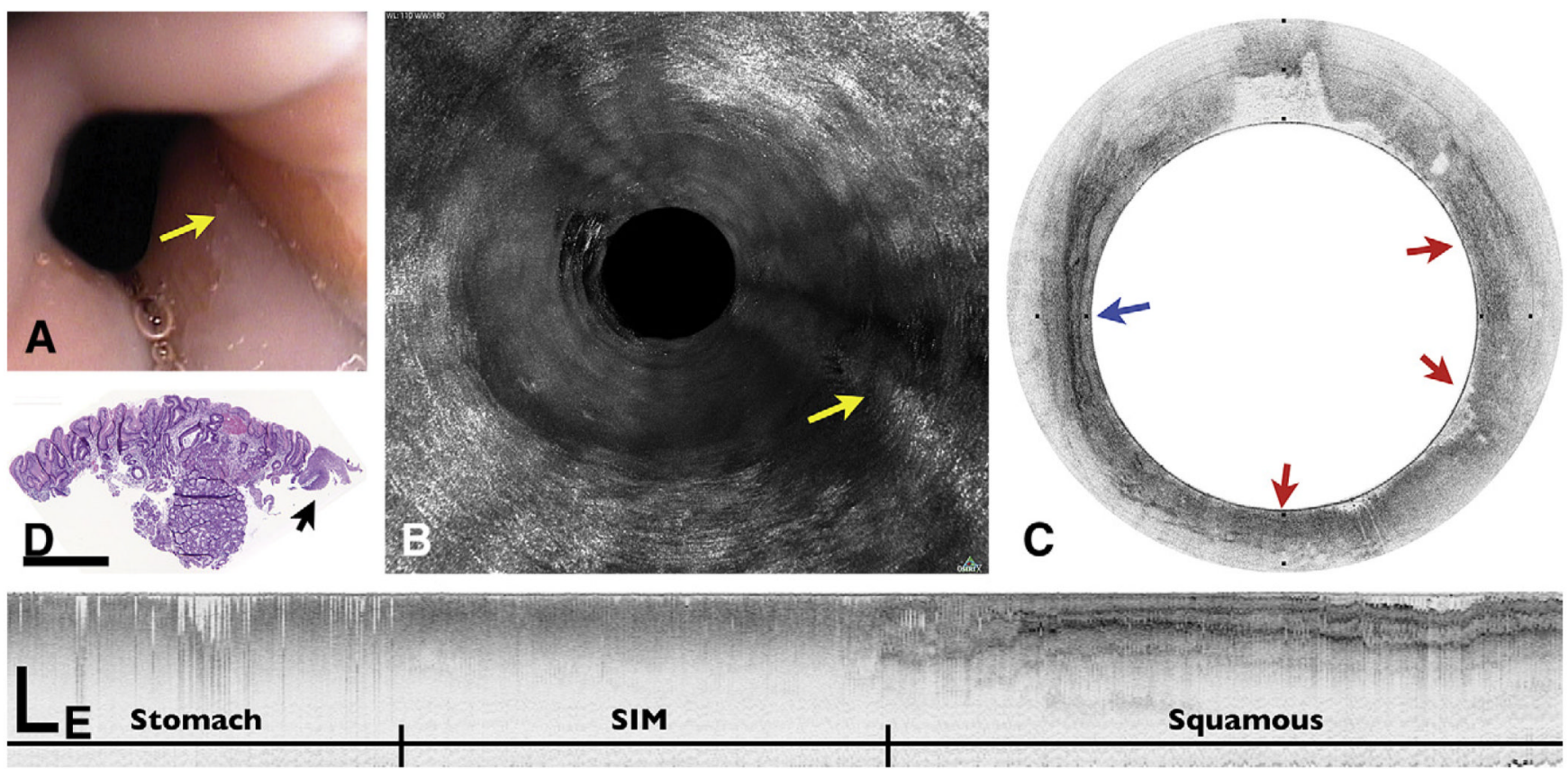

Figure 5.

Presumed Barrett's esophagus. A, Videoendoscopic image shows an irregular SCJ with a tongue of healthy mucosa (arrow). An MIP rendering of the entire volumetric OFDI data set, obtained at the corresponding location in $\mathbf{A}$, enables the visualization of the irregular SCJ including the same lip of squamous mucosa seen in the videoendoscopy image (B, arrow). $\mathbf{C}$, Cross-sectional OFDI image obtained from this location demonstrates squamous mucosa (blue arrow) interspersed with regions that satisfy the OCT criteria for SIM (red arrows). D, Histopathologic image of the biopsy specimen taken from the SCJ consists of gastric cardiactype mucosa, oxyntocardiac-type mucosa, and squamous mucosa with chronic inflammation and no signs of intestinal metaplasia (H\&E, orig. mag. $\times 2$ ). A longitudinal OFDI cross-section through the SCJ shows a 12-mm segment of mucosa that is consistent with SIM. Scale bars and tick marks represent $1 \mathrm{~mm}$. 

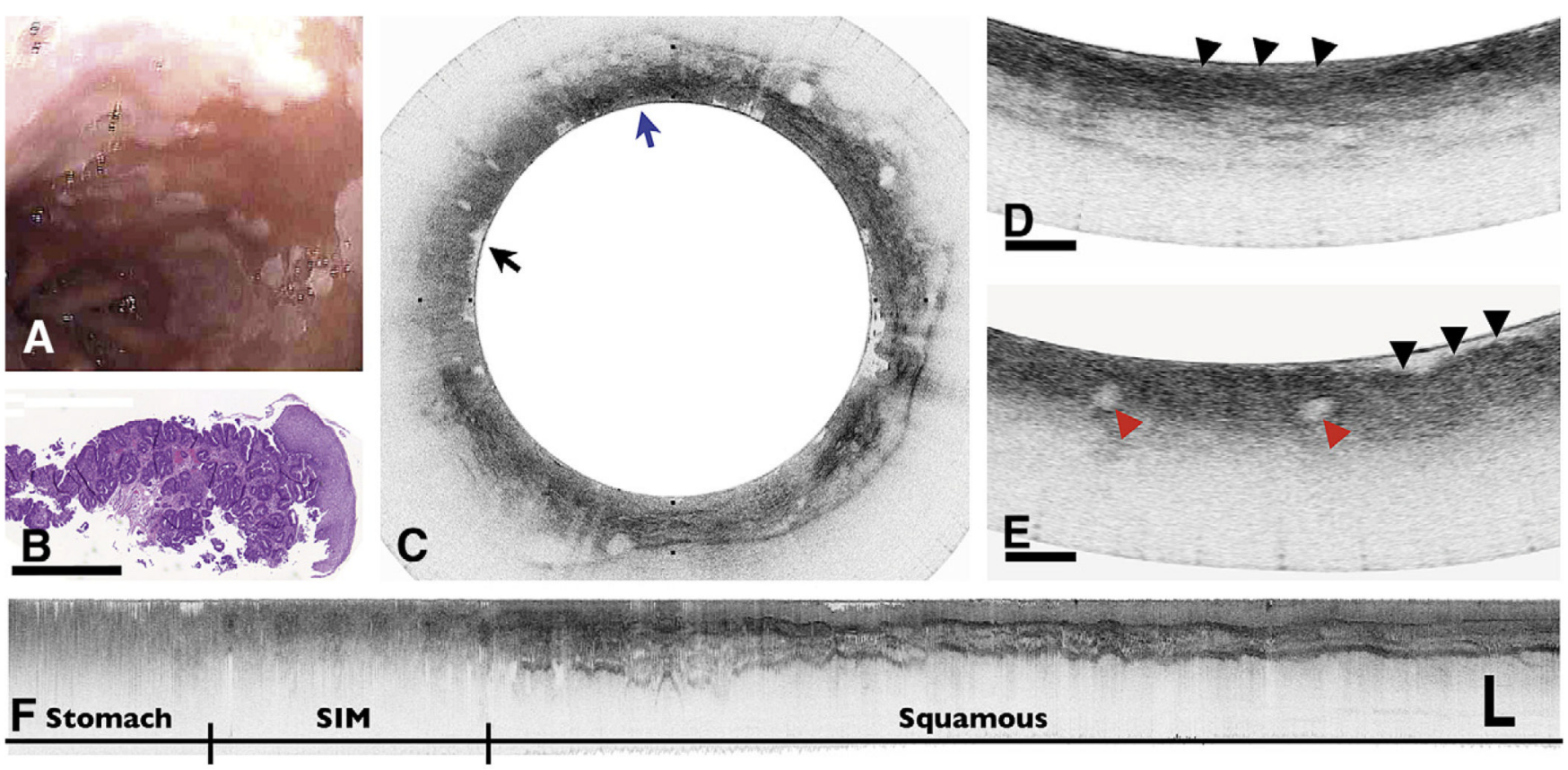

Figure 6.

Barrett's esophagus with dysplasia. A, Videoendoscopic image reveals a patchy ucosa consistent with SIM. B, Histopathologic image of the biopsy specimen taken from the SCJ demonstrates intestinal metaplasia and low-grade dysplasia (H\&E, orig. mag. $\times 2$ ). C, Crosssectional OFDI image demonstrating regions consistent with SIM without dysplasia (blue arrow) and SIM with HGD (black arrow). D, Expanded view of $\mathbf{C}$ taken from the region denoted by the blue arrow in $\mathbf{C}$, demonstrating good surface maturation (arrowheads), which is consistent with SIM without dysplasia. E, Expanded view of $\mathbf{C}$ taken from the region denoted by the black arrow in $\mathbf{C}$, demonstrating features consistent with HGD, including poor surface maturation (black arrowheads) and the presence of dilated glands (red arrowheads) in the mucosa. F, A longitudinal slice highlights the transition from gastric cardia, through a 9-mm segment of SIM and finally into squamous mucosa. Scale bars and tick marks represent $1 \mathrm{~mm}$. 
TABLE 1

OCT diagnostic criteria ${ }^{15,16,18}$

\section{Diagnosis OCT finding}

Squamous mucosa 1. Layered architecture

Cardia $^{*} \quad$ 1. Vertical pit and crypt architecture

2. Highly reflective surface

3. Broad, regular glandular architecture

4. Poor image penetration

$\mathrm{SIM}^{\dagger} \quad$ 1. Lack of layered or vertical pit and crypt architecture

2. Heterogeneous scattering

3. Irregular surface

4. Glands in epithelium with layered architecture

HGD/IMC ${ }^{\dagger} \quad$ 1. Increased surface/subsurface reflectivity (score 0-2)

2. Irregular gland/duct architecture (score 0-2)

See algorithm in reference 12 .

${ }^{\dagger}$ Two of first 3 criteria or criteria 4 indicate SIM.

${ }^{\ddagger}$ Total score of $\geq 2$ indicates HGD/IMC. 